\title{
BthTX-I from Bothrops jararacussu induces apoptosis in human breast cancer cell lines and decreases cancer stem cell subpopulation
}

Patrícia Heloise Alves Bezerra ${ }^{1}$ (D), Isadora Marques Ferreira ${ }^{1}$, Beatriz Tinoco Franceschi ${ }^{1}$, Francine Bianchini ${ }^{1}$, Luciana Ambrósio², Adélia Cristina O. Cintra ${ }^{3}$, Suely Vilela Sampaio ${ }^{3}$, Fabíola Attié de Castro², Maria Regina Torqueti ${ }^{1, *}$

${ }^{1}$ Laboratory of Clinical Cytology, Department of Clinical Analyses, Toxicology and Food Science, School of Pharmaceutical Sciences of Ribeirão Preto, University of São Paulo (USP), Ribeirão Preto, SP, Brazil.

${ }^{2}$ Laboratory of Hematology, Department of Clinical Analyses, Toxicology and Food Science, School of Pharmaceutical Sciences of Ribeirão Preto, University of São Paulo (USP), Ribeirão Preto, SP, Brazil.

${ }^{3}$ Laboratory of Toxinology, Department of Clinical Analyses, Toxicology and Food Science, School of Pharmaceutical Sciences of Ribeirão Preto, University of São Paulo (USP), Ribeirão Preto, SP, Brazil.

\section{Keywords:}

apoptosis

bothropstoxin

breast cancer

cancer stem cells

\begin{abstract}
Background: Breast cancer is the neoplasm with both the highest incidence and mortality rate among women worldwide. Given the known snake venom cytotoxicity towards several tumor types, we evaluated the effects of BthTX-I from Bothrops jararacussu on MCF7, SKBR3, and MDAMB231 breast cancer cell lines.

Methods: BthTX-I cytotoxicity was determined via MTT [3-(4,5-dimethylthiazol-2yl)-2,5-diphenyl-tetrazoliumbromide] assay. Cell death was measured by a hypotonic fluorescent solution method, annexin-V-FITC/propidium iodide staining and by apoptotic/autophagic protein expression. Cancer stem cells (CSCs) were quantified by flow cytometry using anti-CD24-FITC and anti-CD44-APC antibodies and propidium iodide.

Results: BthTX-I at $102 \mu \mathrm{g} / \mathrm{mL}$ induced cell death in all cell lines. The toxin induced apoptosis in MCF7, SKBR3, and MDAMB231 in a dose-dependent manner, as confirmed by the increasing number of hypodiploid nuclei. Expression of pro-caspase 3, pro-caspase 8 and Beclin-1 proteins were increased, while the level of the antiapoptotic protein Bcl-2 was diminished in MCF7 cells. BthTX-I changed the staining pattern of CSCs in MDAMB231 cells by increasing expression of CD24 receptors, which mediated cell death. Conclusions: BthTX-I induces apoptosis and autophagy in all breast cancer cell lines tested and also reduces CSCs subpopulation, which makes it a promising therapeutic alternative for breast cancer.
\end{abstract}

* Correspondence: torqueti@fcfrp.usp.br http://dx.doi.org/10.1590/1678-9199-JVATITD-2019-0010 Received: 22 February 2019; Accepted: 04 June 2019; Published online: 29 July 2019 


\section{Background}

The incidence of cancer has increased over the last few decades, and it has become an evident global public health issue. The International Agency for Research on Cancer of the World Health Organization has predicted 18 million new neoplasm cases for 2018, among which breast cancer figures as the leading tumor type in women [1].

Breast cancer is a highly heterogeneous neoplasm and is classified according to the presence or absence of molecular biomarkers into luminal, HER-2-enriched, and triple-negative subtypes. The luminal subtype expresses hormonal receptors, i.e. estrogen and/or progesterone receptors; the HER-2-enriched subtype expresses neither estrogen nor progesterone receptors, however highly expresses the oncogene HER-2 (human epidermal growth factor receptor type 2); and the triple negative subtype does not express HER-2 or estrogen or progesterone receptors $[2,3]$. These breast cancer subtypes present different clinical outcomes: the luminal cancer responds well to therapy and has a good prognosis, while HER-2-enriched and triple-negative present a bad prognosis.

Molecular subtyping of breast tumors is indispensable because it is directly associated with the therapeutic approach and the patient's prognosis [2]. However, the ineffectiveness and high toxicity of chemotherapeutic drugs, and the fact that they are also associated with tumor resistance have limited breast cancer therapy and promoted a high demand for novel antitumor agents [4]. This has led to research using animal venoms and toxins, that have already demonstrated promising cytotoxic activity against many tumor types such as breast cancer, colorectal cancer, lung adenocarcinoma, melanoma, promyelocytic leukemia [5], chronic myeloid leukemia (CML) [6,7], and myeloproliferative neoplasm [8], both in vitro and in vivo [5].

Burin et al. [6,7] described the antileukemic effects of CRLAAO and LAAO from Bothops pirajai (BpirLAAO-I) in BCRABL1-positive cells lines from CML patients. In addition, the toxin BpirLAAO-I was also able to activate immune cells and lymphocytes of healthy subjects, a process that is relevant for antitumor response in CML patients. Furthermore, BpirLAAO-I induced apoptosis and potentiated the tyronise kinase inhibitor effect on BCR-ABL ${ }^{+}$cells. Additionally, Tavares et al. [8] reported an L-amino-acid oxidase from C. rhodostoma (CR-LAAO) snake venom as a potential antineoplastic agent against HEL 92.1.7 and SET-2 JAK2V617F cell lines derived from myeloproliferative neoplasm patients. Moreover, the cytotoxins CT1 and CT2 from Naja oxiana, CT3 from Naja kaouthia and CT1 from Naja haje showed an important cytotoxicity, mainly mediated by lysosome rupture, against lung adenocarcinoma A549 and promyelocytic leukemia HL60 cells [9].

In this context, the antitumor potential of bothropstoxin I (BthTX-I) was tested. BthTX-I is a phospholipase $\mathrm{A}_{2}\left(\mathrm{PLA}_{2}\right)$ from Bothrops jararacussu venom. BthTX-I, classified as a Lys49-PLA ${ }_{2}$, is catalytically inactive and exerts myotoxic effects through mechanisms that are independent of binding to calcium channels $[10,11]$. BthTX-I has previously presented antitumor activity against HER-2+ breast cancer cells (SKBR3) [12,13].
Thus, the present study evaluated the antitumor potential of BthTX-I against MCF7, SKBR3, and MDAMB231 cell lines, which represent the luminal, HER-2-enriched, and triple-negative breast carcinoma subtypes, respectively.

\section{Methods \\ Cell culture}

The MCF7 (luminal), SKBR3 (HER-2-enriched), and MDAMB231 (triple-negative) breast cancer cell lines were purchased from Rio de Janeiro Cell Bank (BCRJ, Rio de Janeiro, RJ, Brazil) and cultured in RPMI 1640 medium supplemented with $10 \%$ heat-inactivated FBS, $1 \%$ glutamine, $1 \%$ antibiotic/antimycotic solution, and incubated at $37{ }^{\circ} \mathrm{C}$ under $5 \% \mathrm{CO}_{2}$.

\section{Treatment of cell lines}

The cell lines were treated with BthTX-I diluted in estrogen-free RPMI 1640 medium supplemented with charcoal stripped fetal bovine serum (CS-FBS) with increasing concentrations of the toxin $(12,25,51,102,204,409 \mu \mathrm{g} / \mathrm{mL})$. As a positive control, cell lines were treated with one of three chemotherapeutic drugs (cisplatin at $100 \mu \mathrm{M}$, doxorubicin at $4 \mu \mathrm{M}$ or $\mathrm{N}$-desmethyltamoxifen at $20 \mu \mathrm{M})$. For the negative control, cells were treated only with estrogen-free RPMI 1640 medium supplemented with CS-FBS.

\section{Bothropstoxin-I (BthTX-I) purification}

BthTX-I was purified from $B$. jararacussu venom by the Laboratory of Toxinology of the School of Pharmaceutical Sciences of Ribeirão Preto (USP).

B. jararacussu crude venom (150 mg) was fractionated by sizeexclusion chromatography in a Shephacryl S-100 as described by Carone et al. [14]. The eluted fractions was monitored for absorbance at $280 \mathrm{~nm}$, pooled, desalted in a Hi-prep 26/10 desalting column, and lyophilized. The fraction, denominated SPIII, was identified via SDS PAGE by containing phospholipases and myotoxins, which have molecular masses of approximately 14 to $17 \mathrm{kDa}$. For further purification, SPIII was submitted to ion exchange chromatography in a CM Sepharose column ( $40 \mathrm{x}$ $2 \mathrm{~cm}$; Amersham, GE Healthcare Life Science, USA), previously equilibrated with $50 \mathrm{mM}$ ammonium bicarbonate $\mathrm{pH} 8$ (Buffer A). Elution started with the same buffer, followed by a linear gradient of $500 \mathrm{mM}$ ammonium bicarbonate $\mathrm{pH} 8$ (Buffer B). The procedure was performed at a flow rate of $1.5 \mathrm{~mL} / \mathrm{min}$, with fractions of $4 \mathrm{~mL}$ collected based on absorbance at $280 \mathrm{~nm}$. The fraction corresponding to BthTX-I was collected, lyophilized and stored at $4^{\circ} \mathrm{C}$ for subsequent analysis. The purity of BthTX-I was also assayed by $12 \%$ SDS-PAGE and by N-terminal amino-acid sequence, using automated Edman degradation in an automatic protein sequencer (PPSQ 33A, system, Shimadzu).

\section{Cell viability assay}

The cellular viabilities of MCF7, SKBR3 and MDAMB231 were determined via MTT assay, as previously reported [15]. Cells were seeded into 96-well culture plates $\left(2 \times 10^{4}\right.$ cells/well $)$ and 
incubated overnight at $37^{\circ} \mathrm{C}$ under $5 \% \mathrm{CO}_{2}$. The culture medium was removed and cells were treated with BthTX-I (12-409 $\mu \mathrm{g} /$ $\mathrm{mL}$ ) or culture medium (negative control) for $24 \mathrm{~h}$. Twenty $\mu \mathrm{L}$ of a $5 \mathrm{mg} / \mathrm{mL}$ MTT solution was added to each well and plates were incubated for $4 \mathrm{~h}$ at $37^{\circ} \mathrm{C}$, under $5 \% \mathrm{CO}_{2}$. The reaction medium was discarded; the formazan crystals were dissolved in DMSO, and the plates were incubated for $15 \mathrm{~min}$. After shaking for 5 $\mathrm{s}$, the absorbance was recorded at $570 \mathrm{~nm}$ in an Absorbance Microplate Reader Spectramax (Molecular Devices, USA). Cell viability results were expressed as percentages of the negative control. The $\mathrm{IC}_{50}$ values for each cell line were calculated using the software CompuSyn (CompuSyn Inc, USA), and at least three independent experiments were performed.

\section{Apoptosis and necrosis quantification}

The rates of apoptosis and necrosis in MCF7, SKBR3, and MDAMB231 cell lines were quantified using the annexin V-FITC apoptosis detection kit and HFS assays. Annexin V-FITC detection was carried out according to the manufacturer's instructions. Briefly, cells were seeded into 6-well culture plates $\left(2-5 \times 10^{5}\right.$ cells/well), incubated overnight at $37^{\circ} \mathrm{C}$, under $5 \%$ $\mathrm{CO}_{2}$, and further treated with BthTX-I $(102 \mu \mathrm{g} / \mathrm{mL})$ or culture medium (negative control) for $24 \mathrm{~h}$. Next, cells were washed with ice-cold PBS, suspended in $100 \mu \mathrm{L}$ of binding buffer solution $\left(25 \mathrm{mM} \mathrm{CaCl}_{2}, 1.4 \mathrm{M} \mathrm{NaCl}\right.$, and $\left.0.1 \mathrm{M} \mathrm{Hepes} / \mathrm{NaOH}, \mathrm{pH} 7.4\right)$, and incubated in the dark for $30 \mathrm{~min}$ with annexin V-FITC solution (1:100) and PI (1:150) at $100 \mu \mathrm{g} / \mathrm{mL}$. The results of fluorescence-activated cell sorting (FACS) analysis, carried out in a FACS Canto II cytometer, were analyzed using the software FACSDiva (Becton Dickinson, USA). At least three independent tests were performed.

Apoptosis was also assessed using the hypotonic fluorescent solution (HFS) method [16]. Briefly, MCF7, SKBR3, and MDAMB231 cells were cultured in 6-well plates $\left(2-5 \times 10^{5} /\right.$ well $)$ and treated with BthTX-I $(10-200 \mu \mathrm{g} / \mathrm{mL})$, chemotherapeutic drugs (positive control; $4 \mu \mathrm{M}$ doxorubicin, $100 \mu \mathrm{M}$ cisplatin, or $20 \mu \mathrm{M} \mathrm{N}$-desmethyltamoxifen), or culture medium (negative control) for $12 \mathrm{~h}$. Cells were recovered, centrifuged at $240 \times \mathrm{g}$ for $5 \mathrm{~min}$ at $4^{\circ} \mathrm{C}$, and suspended in $200 \mu \mathrm{L}$ of HFS ( $1 \%$ Triton X-100, $1 \%$ sodium citrate, and $100 \mu \mathrm{g} / \mathrm{mL}$ of PI). After a 20 -minute incubation period, cells were analyzed in the Canto II cytometer equipped with the software FACSDiva. The cell death percentage was calculated from the content of genomic DNA, meaning the percentage of hypodiploid nuclei. Five thousand events from the gate were acquired and analyzed by histogram. At least three independent tests were performed.

\section{Bcl-2, Beclin-1 and pro-caspase 3, 8 and 9 levels}

Twenty-four hours after treatment with BthTX-I, MCF7 cells were harvested, washed, and lysed with lysis buffer (Tris $\mathrm{HCl} 20$ $\mathrm{mM} \mathrm{pH} 7.5, \mathrm{NaCl} 150 \mathrm{mM}$, EDTA 1 mM, Igepal CA 630 1\% v/v, sodium pyrophosphate $25 \mathrm{mM}$, sodium orthovanadate $10 \mathrm{mM}$, $\beta$-glycerophosphate $10 \mathrm{mM}$, protease-inhibitor cocktail). Protein concentration in the resulting lysate was determined using Pierce
BCA Protein Quantification kit, as described by the manufacturer. Twenty-five $\mu \mathrm{g}$ of protein were resolved by electrophoresis in $12 \%$ Tris-glycine polyacrylamide gels and transferred to nitrocellulose membranes. The membranes were blocked with $5 \%$ skim milk in TBST $(20 \mathrm{mM}$ Tris- $\mathrm{HCl} \mathrm{pH} \mathrm{7.4,} 150 \mathrm{mM} \mathrm{NaCl}, 0.05 \%$ Tween 20 ), and incubated overnight with the appropriate primary antibody at 1:1,000 or 1:5,000 dilution. The primary antibodies (anti-caspase-3 rabbit polyclonal antibody, anti-caspase-8 mouse monoclonal antibody, anti-human caspase-9 rabbit polyclonal antibody, anti-Bcl-2 rabbit monoclonal antibody and antiBeclin-1 rabbit polyclonal antibody) were purchased from Cell Signaling Technology $y^{\circledR}$ (USA).

After washing, the membranes were incubated with the corresponding horseradish peroxidase-conjugated secondary antibody at 1:7,500 or 1:20,000 dilution in TBST. The secondary antibodies (peroxidase-conjugated AffiniPure anti-rabbit and peroxidase-conjugated AffiniPure anti-mouse) were acquired from Jackson ImmunoResearch (USA). The bound secondary antibody was detected using an Amersham ECL (enhanced chemiluminescence) plus detection reagent, associated with exposure to a light-sensitive film. The actin levels (determined in the same cell lysates) were employed as a protein loading control. The protein expression levels were quantified by densitometry using the software Image J (NIH, version 1.8.0_112) and normalized in relation to actin expression.

\section{CD44 and CD24 immunophenotyping}

Cells were trypsinized, washed with PBS, pelleted by spinning down, and resuspended in PBS. Cells were then incubated with FITC-conjugated mouse anti-human CD44 monoclonal antibody and APC-conjugated mouse anti-human CD24 monoclonal antibody for $30 \mathrm{~min}$, in an ice bath, in the dark, according to the manufacturer's instructions (Becton Dickinson, USA). Next, a PI solution at $100 \mu \mathrm{g} / \mathrm{mL}$ was added to the cells, which were immediately analyzed in the Canto II cytometer equipped with the software FACSDiva. Positive and negative binding beads were used as the positive and negative controls, respectively. The cell subpopulations were calculated among living cells, and at least three independent tests were performed.

\section{Statistical analysis}

Data were analyzed using the software GraphPad Prism 6.0 (GraphPad Software, USA) and expressed as mean \pm standard deviation (SD). Two groups were compared using the Student's $t$ test, while three or more groups were compared by one-way ANOVA combined with the Tukey post-hoc analysis. Statistical significance was indicated by $\mathrm{p}<0.05$.

\section{Results}

\section{Isolation and purification of BthTX-I}

The purification of BthTX-I was successfully carried out by the two chromatographic steps previously mentioned. After the first step, in Sephacryl S100 column, the fraction SPIII (Additional file 1A) 
was identified by means of SDS PAGE containing phospholipase $\mathrm{A}_{2}$ and myotoxins (Additional file 1A, insert). The SPIII fraction was concentrated and subsequently submitted to the CMSepharose column (Additional file 1B). BthTX-I was detected in the fifth fraction, producing a peak found to be highly pure when analyzed by SDS-PAGE (Additional file 1B, insert). BthTX-I was characterized by $\mathrm{N}$-terminal amino-acid sequence obtained by automatic Edman degradation, presenting 20 amino-acid residues (SLFELGKMILQETGKNPAKS) that showed high identity $(100 \%)$ in relation to the $\mathrm{N}$-terminal sequence of the BthTX-I, as reported by Cintra et al. [17].

\section{BthTX-I decreases viability of breast cancer cells}

An MTT assay was used to examine the cytotoxicity of different BthTX-I concentrations towards MCF7, SKBR3, and MDAMB231 cells (Fig. 1). BthTX-I significantly decreased cell viability of MCF7, SKBR3, and MDAMB231 cells at concentrations greater than 102,51 , and $102 \mu \mathrm{g} / \mathrm{mL}$, respectively, $24 \mathrm{~h}$ after exposure. The toxin exerted a concentration-dependent cytotoxic effect only towards SKBR3 cells.

The BthTX-I concentration of $102 \mu \mathrm{g} / \mathrm{mL}$ was selected for subsequent trials because it was the minimum concentration that effectively reduced cell viability of all three cancer cell lines studied when compared with the negative control - it reduced cell viability of MCF7, SKBR3, and MDAMB231 cells by $57.97 \%, 52.82 \%$, and $26.72 \%$, respectively ( $\mathrm{p}<0.05$ ). Moreover, SKBR3 cells were more sensitive to BthTX-I than MCF7 and MDAMB231 cells (Table 1).

\section{BthTX-I induces apoptosis and necrosis of breast cancer cells}

Cell death profile of MCF7, SKBR3, and MDAMB231 cells treated with BthTX-I at $102 \mu \mathrm{g} / \mathrm{mL}$ for $24 \mathrm{~h}$ was determined using the annexin-V and PI staining flow cytometric analysis (Fig. 2). Compared with the negative control (RPMI), BthTX-I significantly induced apoptosis of MCF7 and SKBR3 cells (increase of 65.13\% and $404.45 \%$, respectively; $\mathrm{p}<0.05$ ) and necrosis of MCF7 cells (increase of $86.80 \% ; \mathrm{p}<0.05$ ), but did not induce significant levels of apoptosis or necrosis in MDAMB231 cells.

\section{BthTX-I increases hypodiploid nuclei in breast cancer cells}

To address whether apoptosis induction was the main mechanism of cell death in the breast cancer cell lines in response to BthTX-I, hypodiploid nuclei (apoptotic cells) were quantified using the HFS staining flow cytometric assay (Fig. 3). Twelve hours after BthTX-I treatment at concentrations as low as $10 \mu \mathrm{g} / \mathrm{mL}$, a significantly greater formation of hypodiploid nuclei in MCF7, SKBR3 and MDAMB231 cells was observed when compared with the negative control (increase of $38.20 \%, 8.27 \%$, and $31.13 \%$ respectively; $\mathrm{p}<0.05$ ). The toxin induced apoptosis of the three cell lines in a dose-dependent manner and reached a peak at respective concentrations of 50, 100 and $25 \mu \mathrm{g} / \mathrm{mL}$ in MCF7,

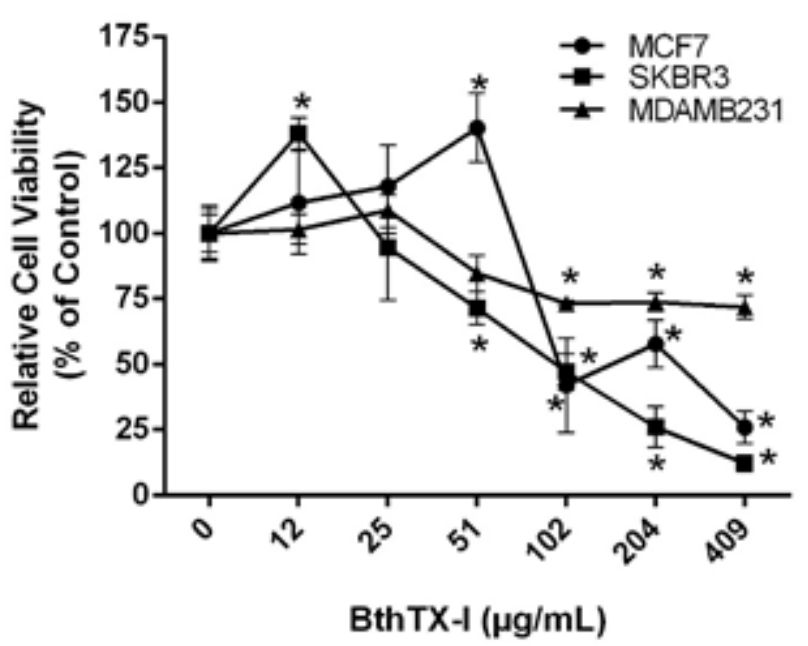

Figure 1. Viability of breast cancer cell lines treated with BthTX-I for 24h, assessed via MTT assay. Values represent the mean \pm SD of at least three independent experiments. ${ }^{*} \mathrm{p}<0.05$ vs. negative control (ANOVA combined with the Tukey post-hoc test).

Table 1. Cytotoxicity of BthTX-I towards breast cancer cell lines.

\begin{tabular}{lc}
\hline Cell line & IC $_{50}(\boldsymbol{\mu g} / \mathbf{m L})$ \\
\hline MCF7 & $104.35 \pm 13.21$ \\
SKBR3 & $81.20 \pm 8.58$ \\
MDAMB231 & $>409 \pm 5.34$ \\
\hline
\end{tabular}

Cells were treated with BthTX-I for $24 \mathrm{~h}$ and cell viability was determined via MTT assay. $I C_{50}$ is the toxin concentration that inhibits cell growth by $50 \%$. Values represent mean $\pm \mathrm{SD}$ of at least three independent experiments.

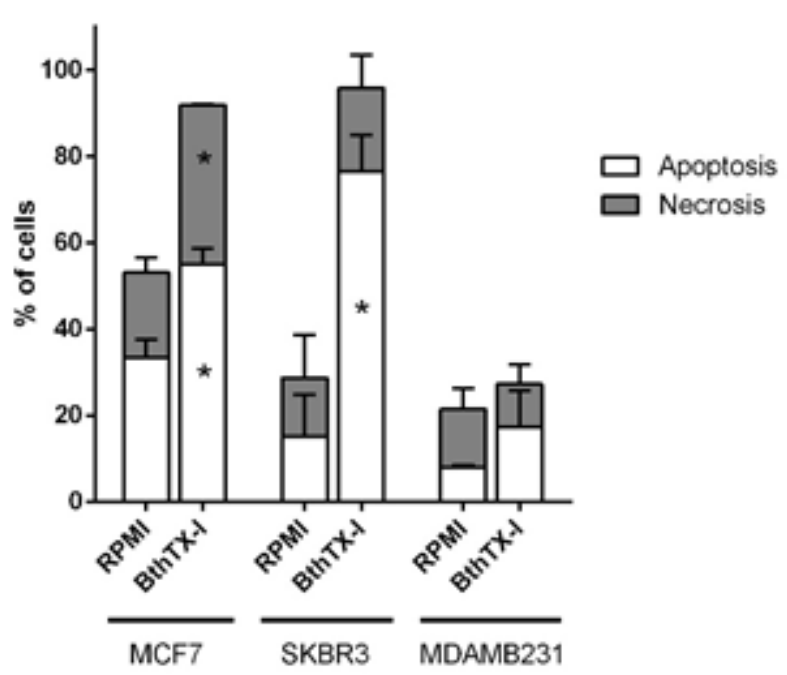

Figure 2. Cell death profile of breast cancer cell lines treated with BthTX-I at $102 \mu \mathrm{g} / \mathrm{mL}$ for $24 \mathrm{~h}$. Cell death was analyzed by the annexin $\mathrm{V}$ and propidium iodide staining flow cytometric method. RPMI: cells incubated in estrogen-free RPMI 1640 medium supplemented with CS-FBS (negative control). Values represent mean $\pm S D$ of at least three independent experiments. ${ }^{*} p<0.05$ vs. negative control (Student's $t$ test). 

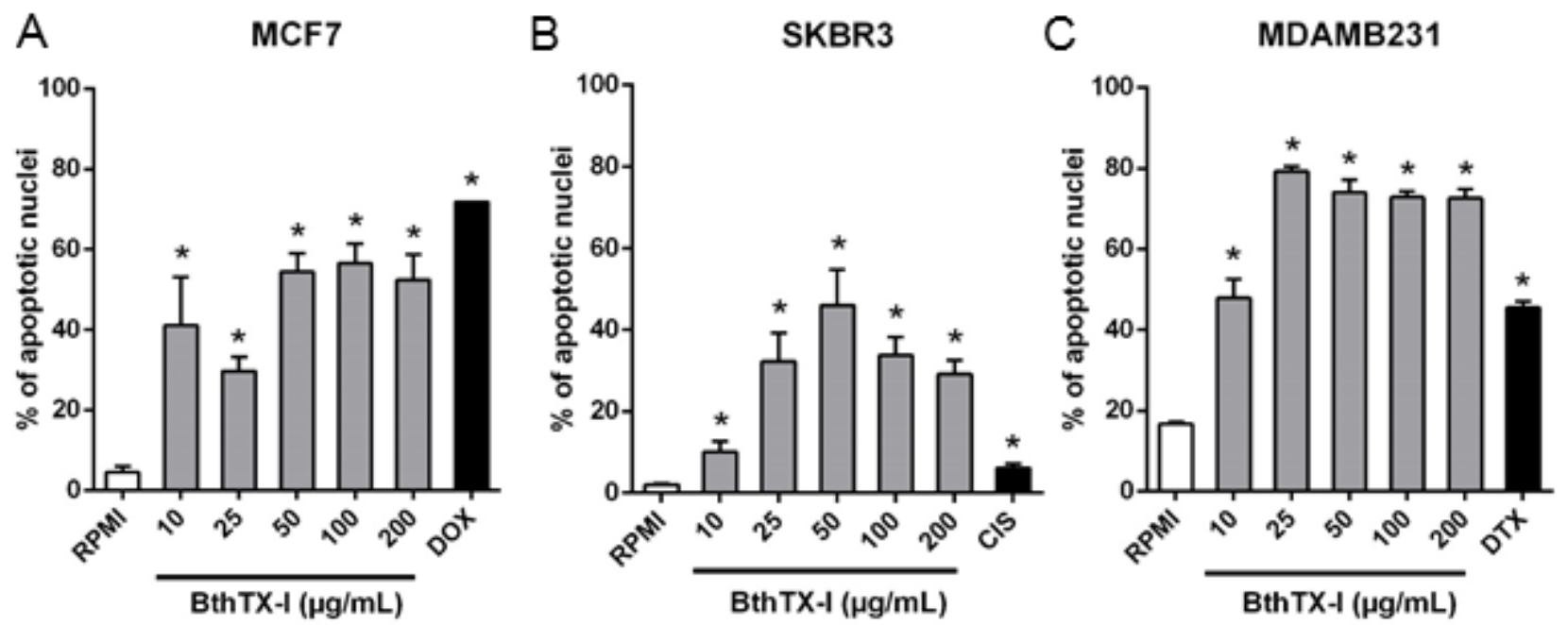

Figure 3. Percentages of hypodiploid nuclei in (A) MCF7, (B) SKBR3, and (C) MDAMB231 breast cancer cell lines treated with BthTX-I for 12h, through quantification of apoptotic nuclei by the hypotonic fluorescent solution method. Cells treated with (A) DOX (4 $\mu$ M doxorubicin), (B) CIS (100 $\mu$ M cisplatin), or (C) DTX (20 $\mu \mathrm{M}$ N-desmethyltamoxifen) were used as positive control. RPMI: cells incubated in estrogen-free RPMI 1640 medium supplemented with CS-FBS (negative control). Values represent mean \pm SD of at least three independent experiments. ${ }^{*} p<0.05$ vs. negative control (ANOVA combined with the Tukey post-hoc test). ns: no significant difference between results in bracket $(p>0.05)$.

SKBR3 and MDAMB231 cells. Therefore, apoptosis induction was the main mechanism by which BthTX-I caused death of breast cancer cells.

\section{BthTX-I strongly induces expression of proapoptotic and proautophagic proteins in MCF7 cells}

The expression levels of proapoptotic (pro-caspase 3, 8 and 9), antiapoptotic (Bcl-2), and proautophagic (Beclin-1) proteins in MCF7 cells treated with BthTX-I at $102 \mu \mathrm{g} / \mathrm{mL}$ for $24 \mathrm{~h}$ were quantified by Western Blot analysis (Fig. 4). Compared with the negative control, BthTX-I upregulated the expression of procaspase 3, pro-caspase 8 and of Beclin-1 (increase of 83.94\%, $535 \%$, and $29.57 \%$, respectively). In addition, the toxin also downregulated the expression of $\mathrm{Bcl}-2$ (reduction of 99.97\%). BthTX-I did not alter the levels of pro-caspase 9 expression. Doxorubicin was used as positive control for apoptosis and autophagy induction.

\section{CD44/CD24 expression levels on breast cancer cell lines treated with BthTX-I}

To identify the cancer stem cell (CSC) subpopulation, we analyzed expression of the markers CD44 and CD24 by flow cytometry (Fig. 5). First, the breast carcinoma cell lines were characterized as $\mathrm{CD} 44^{+} / \mathrm{CD} 24^{- \text {llow }}$. Second, analysis of this subpopulation among the three cell lines revealed that the MDAMB231 cell line presented the greatest CSC prevalence (99.3\%) when compared with the MCF7 and SKBR3 cell lines ( $4.5 \%$ and $0.55 \%$, respectively) (Fig. 5A). Compared with the negative control, treatment with BthTX-I changed the expression pattern of a significant number of MDAMB231 cells $(9.08 \%$ cells; $\mathrm{p}<$

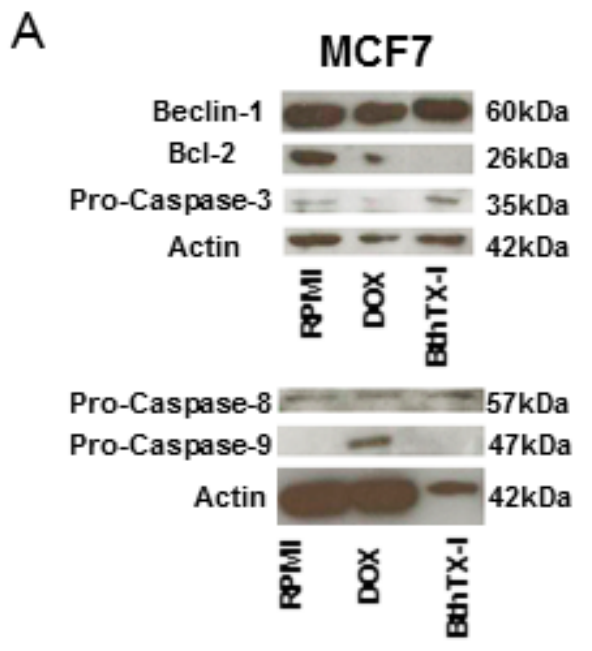

B

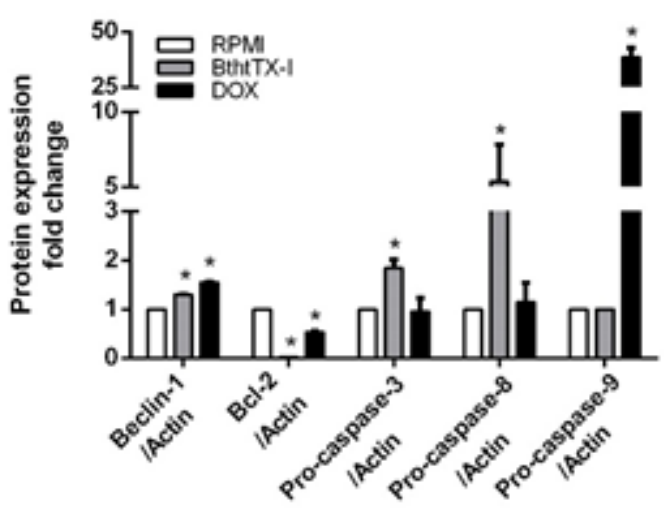

Figure 4. Beclin-1 and Bcl-2 protein (A) expression and (B) quantification in MCF7 cells treated with BthTX-I at $102 \mu \mathrm{g} / \mathrm{mL}$ for 24h. DOX: cells treated with doxorubicin at $4 \mu \mathrm{M}$ (positive control). RPMl: cells incubated in estrogenfree RPMI 1640 medium supplemented with CS-FBS (negative control). ${ }^{*} \mathrm{p}<$ 0.05 vs negative control (Student's $t$ test). 
A

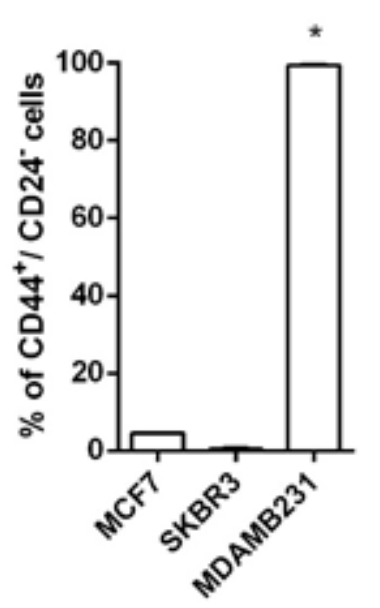

B MDAMB231

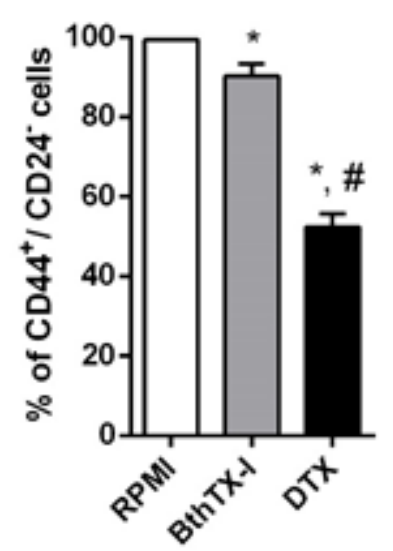

MDAMB231

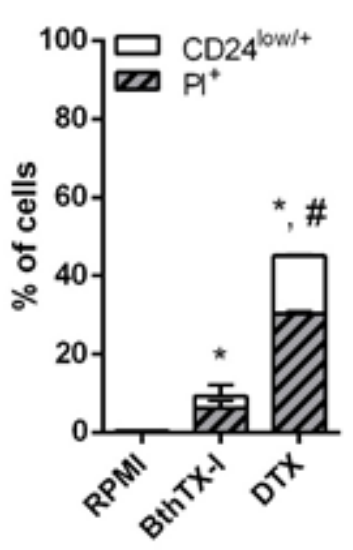

Figure 5. Quantification of cancer stem cells. The CSCs immunophenotyping quantification was carried out by flow cytometry using propidium iodide staining of the biomarkers CD24 and CD44. (A) Percentage of CD44 /CD24- cancer stem cells in untreated breast cancer cell lines. (B) Percentage of CD44 /CD24/low cancer stem cells in the MDAMB231 cell population treated with BthTX-I at $102 \mu \mathrm{g} / \mathrm{mL}$ for $24 \mathrm{~h}$. (C) Expression of CD24 cells in the cell population depicted in (B). DTX: cells treated with $20 \mu \mathrm{M}$ N-desmethyltamoxifen (positive control). RPMl: cells incubated in estrogen-free RPMI 1640 medium supplemented with CS-FBS (negative control). PI: Propidium iodide staining. Values represent mean \pm SD of at least three independent experiments. $p<0.05$ vs. *negative control and vs. "BthTX-I (Student's $t$ test).

$0.05)$ from $\mathrm{CD} 44^{+} / \mathrm{CD} 24^{-}$to $\mathrm{CD} 44^{+} / \mathrm{CD} 24^{\text {low }}$ (Fig. 5B). Although $\mathrm{CD} 44^{+} / \mathrm{CD} 24^{\text {low }}$ cells are still classified as CSCs, most of them became $\mathrm{PI}^{+}$(6.23\%) (Fig. 5C), indicating that this alteration in CD24 expression resulted in cell death. We used binding beads and $\mathrm{N}$-desmethyltamoxifen as the CD24 binding control and positive control, respectively (Additional file 2).

\section{Discussion}

Previous studies have demonstrated the cytotoxicity of snake venoms towards several tumor types $[5,18]$. This property is attributed to the phospholipase $\mathrm{A}_{2}\left(\mathrm{PLA}_{2}\right)$ class of enzymes, independently of their catalytic capability. Some $\mathrm{PLA}_{2} \mathrm{~s}$ from snakes belonging to the genus Bothrops exert antineoplastic action, such as myotoxin-II from Bothrops asper that is cytotoxic to adrenal tumor, and MjTX-II from Bothrops moojeni venom that is cytotoxic to Ehrlich ascites tumor, SKBR3 breast adenocarcinoma, and Jurkat T-cell leukemia [19]. Furthermore, a prior study found that MjTX-I, a PLA from Bothrops moojeni, is able to diminish cell viability of chronic myeloid leukemia cells (K562-S and K562-R $\mathrm{BCR}-\mathrm{ABL}^{+}$) and induce apoptosis through activation of Caspases 3, 8 and 9 [20]. It remains unclear exactly how bothropstoxins from $B$. jararacussu affect mammary carcinomas.

Gebrim et al. [13] have shown that BthTX-I is cytotoxic to $75-90 \%$ of B16F10 melanoma cells, Jurkat T-cell leukemia cells, and in vivo solid tumor (S180), but to only $45 \%$ of SKBR3 breast cancer cells [13]. Moreover, the same study found that a BthTX-I modified with $p$-bromophenacyl bromide (BPB-BthTX-I) and with a peptide synthesized from the C-terminal part of this toxin (pep-BthTX-I), exhibited an increased cytotoxicity to cancer cells and reduced myotoxicity [13]. Our findings show that BthTX-I at $102 \mu \mathrm{g} / \mathrm{mL}$ strongly decreased cell viability of SKBR3 cells $(52.82 \%)$, which corroborates the finding of Gebrim et al. [13]. Herein, we have also reported the cytotoxicity of BthTX-I towards luminal (MCF7) and triple-negative (MDAMB231) breast cancer cell line subtypes (with respective decreases in cell viability of $57.98 \%$ and $26.72 \%$ ).

It is well known that apoptosis happens when cell death is genetically programed and not accidental [21,22]. In general, it is mediated by the action of genes and proteins that activate effector caspases (cysteine-dependent aspartate-specific proteases), and occurs through pathways that do not elicit an inflammatory response. As apoptosis is an innate antitumor mechanism, it is a limiting factor in neoplastic diseases so that its induction is a target of many chemotherapeutic drugs [21,22].

The present study demonstrated that the antineoplastic activity of BthTX-I is mainly mediated by apoptosis induction. The BthTX-I in MCF7 cells promoted phosphatidylserine externalization and hypodiploid nuclei formation, as well as augmented expression of two pro-apoptotic proteins (procaspases 3 and 8) and diminished expression of the anti-apoptotic protein $\mathrm{Bcl}-2$. These findings corroborate previous reports that BthTX-I induces apoptosis in HL-60, human promyelocytic leukemia cell line and liver carcinoma cells (HepG2) [23]. Furthermore, other PLA toxins from snakes of the genus Bothrops may trigger apoptosis of tumor cells, such as BnSP-6 from Bothrops pauloensis, which elicits phosphatidylserine externalization, upregulates expression of the pro-apoptotic caspase 8 gene and downregulates expression of the antiapoptotic Bcl-2, Bcl211, and BIRC-5 genes in MDAMB231 triple- 
negative breast cancer cells [24]. Additionally, in contrast to a report in the literature that MCF7 cells do not express caspase 3 [25], our findings suggest that BthTX-I can restore pro-caspase 3 expression and re-sensitize MCF7 cells to apoptosis, since this type of restoration was previously observed after exposure to another natural compound, curcumin [26].

Autophagy differs from apoptosis with respect to cell morphology and is characterized by degradation of cellular components and formation of vacuoles [21]. The initial autophagy stage is mediated by the emergence of membranes involved in the targeting of cytoplasm portions and organelles to form autophagosomes, which fuse with lysosomes; lysosomal enzymes degrade the autophagosome contents. It is well described in the literature that autophagy depends on the proteins Atg6 (Beclin-1), Atg5 and Atg7 [27]. As BthTX-I increased Beclin-1 expression, we concluded that this toxin induced autophagy conjointly with apoptosis in the breast cancer cell lines herein investigated. These results are in accord with literature reports that some PLA $\mathrm{P}_{2}$, such as BnSP- 6 from Bothrops pauloensis, stimulate autophagy pathways that induce the formation of autophagic vacuoles in triple-negative breast cancer cells (MDAMB231) [24], and that crotoxin stimulates the formation of autophagic vacuoles in luminal breast cancer cells (MCF7) [28].

In breast tumors, the cancer stem cells (CSC) can be identified by the expression of $\mathrm{CD} 44$ and by low or absent expression of $\mathrm{CD} 24\left(\mathrm{CD} 44^{+} / \mathrm{CD} 24^{- \text {llow }}\right)[29,30]$. CSCs are usually associated with resistance to chemotherapy and neoplasm relapse [31]. We found that most of the triple-negative breast cancer cells (MDAMB231) behaved as CSCs, while luminal (MCF7) and HER2-enriched (SKBR3) cell populations did not show an expressive CSC subpopulation. This result corroborates the findings reported by Ricardo et al. [32] and reinforces the high aggressiveness of triple-negative breast cancer in the clinic. Additionally, our $\mathrm{IC}_{50}$ results showed that BthTX-I is more efficient in diminishing cell viability in HER-2-enriched breast cancer subtype, followed by luminal and then by triple-negative. Thus, we speculate that the lessened effect of BthTX-I on triple-negative subtype is attributable to the higher aggressiveness of this tumor.

We also found that treatment of MDAMB231 cells with BthTX-I altered the CSC staining pattern by increasing CD24 expression, which in turn reduced cell viability. The literature reports that CD24 expression is lower in progenitor cells than in differentiated cells. Although some authors state that the presence of CD24 promotes cell proliferation and survival, other authors conclude that the presence of CD24 suppresses invasion and metastasis [33].

The findings reported herein also suggest that BthTX-I promotes autophagy by upregulating expression of Beclin-1 in MCF7 cells. It has already been demonstrated that C2 ceramide, a known autophagy inductor, decreases the $\mathrm{CD} 44^{+} / \mathrm{CD} 24^{- \text {-low }}$ cell subpopulation in breast cancer cells (MCF7) and larynx cancer cells (Hep-2), indicating that activation of this cell-death pathway may be a mechanism to diminish the cancer-cell subpopulation that is resistant to conventional therapy [34].

Taken together, ours results suggest that BthTX-I exerts a noticeable cytotoxicity towards luminal (MCF7), HER-2enriched (SKBR3), and triple-negative (MDAMB231) breastcancer-cell lines. This toxin not only induces cell death mainly via autophagy and the apoptosis pathway, but also decreases the number of MDAMB231 CSC - which are associated with resistance to chemotherapeutic drugs, cancer relapse, and aggressiveness of triple-negative neoplasms. Therefore, BthTX-I is a promising candidate for therapy against breast tumors that deserves to be tested in vivo.

\section{Acknowledgments}

The authors would like to thank Mrs. Fabiana Rosseto de Morais for FACS experiments, Mr. Thiago Abrahão Silva for technical support and toxin purification and the University of São Paulo (USP, Brazil) for providing the laboratory facilities at the Laboratory of Clinical Cytology and Laboratory of Toxinology.

\section{Abbreviations}

HER-2: human epidermal growth factor receptor-type 2; BthTX-I: bothropstoxin I; PLA 2 : phospholipase $\mathrm{A}_{2}$; RPMI-1640: Roswell Park Memorial Institute 1640; FBS: fetal bovine serum; CML: chronic myeloid leucemia; CS-FBS: charcoal-stripped fetal bovine serum; MTT: 3-(4,5-dimethylthiazol-2-yl)-2,5diphenyl-tetrazoliumbromide; DMSO: dimethyl sulfoxide; PI: propidium iodide; FACS: fluorescence-activated cell sorting; HFS: hypotonic fluorescent solution; SD: standard deviation; $\mathrm{IC}_{50}$ : half maximal inhibitory concentration; CSC: cancer stem cells; Caspase: cystein-dependent aspartate-specific protease.

\section{Availability of data and materials}

Not applicable

\section{Funding}

The present study was supported by the following Brazilian agencies: São Paulo Research Foundation (FAPESP - grant $\mathrm{n}$. 2011/23236-4), the Coordination for the Improvement of Higher Education Personnel (CAPES - grant n. 88882.180867/201801) and National Council for Scientific and Technological Development (CNPq - grant n. 168880/2018-0 and n. 305163/2015-9).

\section{Competing interests}

The authors declare that they have no competing interests.

\section{Authors' contributions}

PHAB, FB, and MRT conceived and designed the experiments. SVS provided the toxin that was purified in the Laboratory of Toxinology. PHAB, IMF, BTF, and FB carried out the 
experiments. $\mathrm{PHAB}, \mathrm{LA}$, and $\mathrm{FAC}$ analyzed and interpreted data and statistics. SVS and ACOC analyzed purification data and drafted the manuscript. PHAB, FAC and MRT interpreted the results and drafted the manuscript. All authors read and approved the final manuscript.

\section{Ethics approval}

Not applicable.

\section{Consent for publication}

Not applicable.

\section{Supplementary material}

The following online material is available for this article:

\begin{abstract}
Additional file 1. Isolation of BthTX-I from Bothrops jararacussu venom. (A) Chromatographic profile of $B$. jararacussu crude venom (150 mg) on Sephacryl S 100 column under elution with $20 \mathrm{mM}$ Tris $\mathrm{Hcl}+150 \mathrm{mM} \mathrm{NaCl}$, $\mathrm{pH}$ 8. Fraction of $1 \mathrm{~mL}$ was collected at a flow rate of 12 $\mathrm{mL} / \mathrm{h}$, at room temperature. Inset: $12 \%$ SDS- PAGE of SPIII fraction under reducing conditions (1); molecular mass standards (2). Purification of BthTX-I. (B) Chromatography of $20 \mathrm{mg}$ of SPIII fraction on CM-Sepharose previously equilibrated with $50 \mathrm{mM}$ ammonium bicarbonate, $\mathrm{pH} 8$, and then eluted on a concentration gradient of up to 50 $\mathrm{mM}$ of the same buffer. Fraction of $4 \mathrm{~mL}$ was collected at a flow rate of $1.52 \mathrm{~mL} / \mathrm{min}$, at room temperature. Inset: $12 \%$ SDS- PAGE of BthTX-I under reducing conditions (2); molecular mass standards (1).
\end{abstract}

Additional file 2. Flow cytometric analysis of cancer stem cell subpopulation in MDAMB231 cells treated with BthTX-I at $102 \mu \mathrm{g} / \mathrm{mL}$ for $24 \mathrm{~h}$. The CD24 and CD44 markers were quantified and antibody testing was performed with Beads (control). DTX: N-desmethyltamoxifen at $20 \mu \mathrm{M}$ (positive control). RPMI: cells incubated in estrogen-free RPMI 1640 medium supplemented with CS-FBS (negative control).

\section{References}

1. Globocan, larc. OMS. Cancer today. Breast cancer: Estimated incidence, mortality and prevalence worldwide in 2018. 2018.

2. Makki J. Diversity of breast carcinoma: histological subtypes and clinical relevance. Clin Med Insights Pathol. 2015;8:23-31.

3. Dai X, Xiang L, Li T, Bai Z. Cancer hallmarks, biomarkers and breast cancer molecular subtypes. J Cancer. 2016;7(10):1281-94.

4. Chang M. Tamoxifen resistance in breast cancer. Biomol Ther. 2012;20(3):256-67.

5. Calderon LA, Sobrinho JC, Zaqueo KD, de Moura AA, Grabner AN, Mazzi MV, et al. Antitumoral activity of snake venom proteins: new trends in cancer therapy. Biomed Res Int. 2014;2014:203639.

6. Burin SM, Ghisla S, Ouchida AT, Aissa AF, Coelho MG, Costa TR, et al. CR-LAAO anitleukemic effect against $\mathrm{Bcr}-\mathrm{Abl}(+)$ cells is mediated by apoptosis and hydrogen peroxidase. Int J Biol Macromol. 2016;86:309-20.
7. Burin SM, Ayres LR, Neves RP, Ambrósio L, de Morais FR, Dias-Baruffi M, et al. L-amino acid oxidase isolated from Bothrops pirajai induces apoptosis in BCR-ABL-positive cells and potentiates imatinib mesylate effect. Basic Clin Pharmacol Toxicol. 2013;113(2):103-12.

8. Tavares C, Maciel T, Burin S, Ambrósio L, Ghisla S, Sampaio S, et al. L-Amino acid oxidase isolated from Calloselasma rhodostoma snake venom induces cytotoxicity and apoptosis in JAK2V617F-positive cell lines. Rev Bras Hematol Hemoter. 2016;38(2):128-34.

9. Feofanov AV, Sharonov GV, Astapova MV, Rodionov DI, Utkin YN, Arseniev AS. Cancer cell injury by cytotoxins from cobra venom is mediated through lysosomal damage. Biochem J. 2005;390(Pt 1):11-8.

10. Gutiérrez JM, Lomonte B. Phospholipase A2 myotoxins from Bothrops snake venoms. Toxicon. 1995;33(11):1405-24.

11. Andrião-Escarso SH, Soares AM, Rodrigues VM, Angulo Y, Díaz C, Lomonte B, et al. Myotoxic phospholipases A2 in Bothrops snake venoms: effect of chemical modifications on the enzymatic and pharmacological properties of bothropstoxins from Bothrops jararacussu. Biochimie. 2000;82(8):755-63.

12. Roberto PG, Kashima S, Marcussi S, Pereira JO, Astolfi-Filho S, Nomizo $A$, et al. Cloning and identification of a complete cDNA coding for a bactericidal and antitumoral acidic phospholipase A2 from Bothrops jararacussu venom. Protein J. 2004;23(4):273-85.

13. Gebrim LC, Marcussi S, Menaldo DL, de Menezes CS, Nomizo A, Hamaguchi A, et al. Antitumor effects of snake venom chemically modified Lys 49 phospholipase A2-like BthTX-I and a synthetic peptide derived from its C-terminal region. Biologicals. 2009;37(4):222-9.

14. Carone SEI, Costa TR, Burin SM, Cintra ACO, Zoccal KF, Bianchini FJ, et al. A new I-amino acid oxidase from Bothrops jararacussu snake venom: Isolation, partial characterization, and assessment of pro-apoptotic and antiprotozoal activities. Int J Biol Macromol. 2017;103:25-35.

15. Mosmann T. Rapid colorimetric assay for cellular growth and survival: application to proliferation and cytotoxicity assays. J Immunol Methods. 1983;65(1-2):55-63.

16. Nicoletti L, Migliorati G, Pagliacci MC, Grignani F, Riccardi C. A rapid and simple method for measuring thymocyte apoptosis by propidium iodide staining and flow cytometry. J Immunol Methods. 1991;139(2):271-9.

17. Cintra AC, Marangoni S, Oliveira B, Giglio JR. Bothropstoxin-I: amino acid sequence and function. J Protein Chem. 1993;12(1):57-64.

18. Vyas VK, Brahmbhatt K, Bhatt H, Parmar U. Therapeutic potential of snake venom in cancer therapy: current perspectives. Asian Pac J Trop Biomed. 2013;3(2):156-62.

19. Rodrigues RS, Izidoro LF, de Oliveira RJJr, Sampaio SV, Soares AM, Rodrigues VM. Snake venom phospholipases A2: a new class of antitumor agents. Protein Pept Lett. 2009;16(8):894-8.

20. Benati RB, Costa TR, Cacemiro MC, Sampaio SV, Castro FA, Burin SM. Cytotoxic and pro-apoptotic action of MjTX-I, a phospholipase A2 isolated from Bothrops moojeni snake venom, towards leukemic cells. J Venom Anim Toxins incl Trop Dis. 2018;24.

21. Grivicich I, Regner A, Rocha AB. Morte celular por apoptose. Rev Bras Cancerol. 2007;53(3):335-43.

22. Fulda S, Gorman AM, Hori O, Samali A. Cellular stress responses: cell survival and cell death. Int J Cell Biol. 2010;2010:214074.

23. Silva CP, Costa TR, Paiva RMA, Cintra ACO, Menaldo DL, Antunes LMG, et al. Antitumor potential of the myotoxin BthTX-I from Bothrops jararacussu snake venom: evaluation of cell cycle alterations and death mechanisms induced in tumor cell lines. J Venom Anim Toxins incl Trop Dis. 2015;21:44.

24. Azevedo FV, Lopes DS, Cirilo Gimenes SN, Achê DC, Vecchi L, Alves PT, et al. Human breast cancer cell death induced by BnSP-6, a Lys-49 PLA 2 homologue from Bothrops pauloensis venom. Int J Biol Macromol. 2016;82:671-7.

25. Jänicke RU. MCF-7 breast carcinoma cells do not express caspase-3. Breast Cancer Res Treat. 2018;117(1):219-21.

26. Karimpour M, Feizi MAH, Mahdavi M, Krammer B, Verwanger T, Najafi F, et al. Development of curcumin-loaded gemini surfactant nanoparticles: 
Synthesis, characterization and evaluation of anticancer activity against human breast cancer cell lines. Phytomedicine. 2019;57:183-90.

27. Kang R, Zeh HJ, Lotze MT, Tang D. The Beclin 1 network regulates autophagy and apoptosis. Cell Death Differ. 2011;18(4):571-80.

28. Yan C, Yang YP, Qin ZH, Gu ZL, Reid P, Liang ZQ. Autophagy is involved in cytotoxic effects of crotoxin in human breast cancer cell line MCF-7 cells. Acta Pharmacol Sin. 2007;28(4):540-8.

29. Al-Hajj M, Wicha MS, Benito-Hernandez A, Morrison SJ, Clarke MF. Prospective identification of tumorigenic breast cancer cells. Proc Natl Acad Sci U S A. 2003;100(7):3983-8.

30. Oliveira LR, Jeffrey SS, Ribeiro-Silva A. Stem cells in human breast cancer Histol Histopathol. 2010;25(3):371-85.
31. Beck B, Blanpain C. Unravelling cancer stem cell potential. Nat Rev Cancer. 2013;13(10):727-38.

32. Ricardo S, Vieira AF, Gerhard R, Leitão D, Pinto R, Cameselle-Teijeiro JF, et al. Breast cancer stem cell markers CD44, CD24 and ALDH1: expression distribution within intrinsic molecular subtype. J Clin Pathol. 2011;64(11):937-46

33. Jaggupilli A, Elkord E. Significance of CD44 and CD24 as cancer stem cell markers: an enduring ambiguity. Clin Dev Immunol. 2012;2012:708036.

34. Nami B, Donmez $\mathrm{H}$, Koçak N. Autophagy reduces subpopulation of CD44C/CD24/low phenotype cancer stem cells in MCF7 and Hep-2 cells culture. J Cancer Stem Cell Res. 2015;3:e1002. 